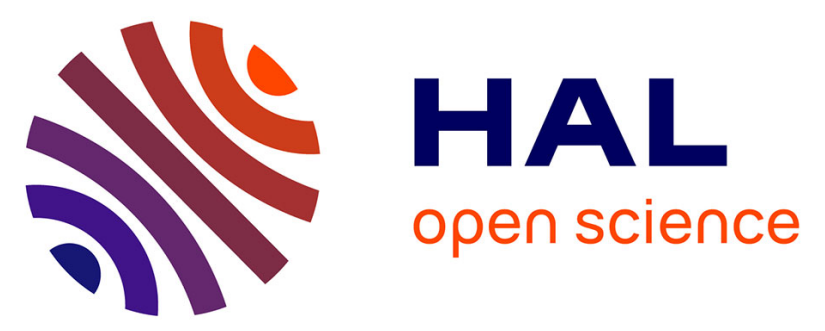

\title{
Ask and you might receive: The actor-partner interdependence model approach to estimating cultural and gender variations in social support
}

Biru Zhou, Dara Heather, Alessia Di Cesare, Andrew G. Ryder

\section{- To cite this version:}

Biru Zhou, Dara Heather, Alessia Di Cesare, Andrew G. Ryder. Ask and you might receive: The actor-partner interdependence model approach to estimating cultural and gender variations in social support. European Journal of Social Psychology, 2017, 47 (4), pp.412-428. 10.1002/ejsp.2251 . hal01626449

\author{
HAL Id: hal-01626449 \\ https://hal.science/hal-01626449
}

Submitted on 2 Nov 2017

HAL is a multi-disciplinary open access archive for the deposit and dissemination of scientific research documents, whether they are published or not. The documents may come from teaching and research institutions in France or abroad, or from public or private research centers.
L'archive ouverte pluridisciplinaire HAL, est destinée au dépôt et à la diffusion de documents scientifiques de niveau recherche, publiés ou non, émanant des établissements d'enseignement et de recherche français ou étrangers, des laboratoires publics ou privés. 
Ask and You Might Receive: The Actor-Partner Interdependence Model Approach to Estimating Cultural and Gender Variations in Social Support

\author{
Biru Zhou \\ Université de Montéal \\ Dara Heather \\ Concordia University \\ Alessia Di Cesare \\ Concordia University \\ Andrew G. Ryder \\ Concordia University and Jewish General Hospital
}

\begin{abstract}
Author Note
Corresponding authors: Biru Zhou, Ph.D. (biru.zhou@gmail.com or biru.zhou@umontreal.ca; 1(514)343-6111, ext. 55721; Fax: 1(514)343-2334), and Andrew G. Ryder, Ph.D. (andrew.ryder@concordia.ca; 1(514) 848-2424 ext. 5379).

Acknowledgements: The authors wish to thank Dr. David A. Kenny and Dr. Tom Loeys for their valuable advice and suggestions on the analysis of the Actor-Partner Interdependence Model used in this study.
\end{abstract}




\begin{abstract}
As an essential part of close relationships, social support is a dynamic interactive process. This paper aims to simultaneously investigate social support seeking and provision behaviours using the Actor-Partner Interdependence Model (APIM). 92 friendship dyads participated in this study. Supportive vs. negative friendship qualities were used to predict different support seeking and support provision behaviours during an experimental task. Cultural and gender variations were also examined. Results showed that self-reported friendship qualities influence support seeking and provision behaviours intrapersonally and interpersonally. Female participants were more likely to provide emotion-focused support than were male participants. After accounting for friendship qualities in the dyads, there was no evidence of cultural group differences on support seeking or provision behaviours among same-sex friends. These results demonstrate the conceptual and empirical advantages of using APIM to unpack cultural and gender variations in social support processes.

Keywords: Social Support, Gender, Friendship, Actor-Partner Interdependence Model, Cultural Contexts
\end{abstract}


Ask and You Might Receive: The Actor-Partner Interdependence Model Approach to Estimating Cultural and Gender Variations in Social Support

When faced with loss, disappointment and life changing challenges, a helping hand from friends and family is both welcomed and expected. Social support has been examined in a variety of disciplines, such as anthropology, epidemiology, community health, and psychology (e.g., Cable, Bartley, Chandola, \& Sacker, 2013; Goldsmith, 2004; Jacobson, 1987; H. S. Kim, Sherman, \& Taylor, 2008), and has been shown to buffer a range of mental and physical health problems (e.g., Bolger, Zuckerman, \& Kessler, 2000; Masters, Stillman, \& Spielmans, 2007; Wonderlich-Tierney \& Vander Wal, 2010). The well-known positive effects of social support include reductions in social anxiety and eating disorder symptoms (Wonderlich-Tierney \& Vander Wal, 2010), depressed mood (Bolger et al., 2000; Schuster, Kessler, \& Aseltine, 1990), and severity of chronic stressful physical conditions (Martin, Davis, Baron, Suls, \& Blanchard, 1994; Masters et al., 2007). The most frequently identified sources of social support include family, friends and romantic partners (Carbery \& Buhrmester, 1998; Day \& Livingstone, 2003; Zimet, Dahlem, Zimet, \& Farley, 1988).

Although spousal support has usually been in the spotlight in the social support literature (e.g., Bolger et al., 2000; Bolger \& Amarel, 2007; Cutrona, Shaffer, Wesner, \& Gardner, 2007; Don, Mickelson, \& Barbee, 2013; Verhofstadt, Buysse, \& Ickes, 2007; Verhofstadt, Lemmens, \& Buysse, 2012; Wang \& Repetti, 2014), support from non-kin friends has also garnered some scholarly attention (e.g., Crocker \& Canevello, 2008; Day \& Livingstone, 2003; Deci, La Guardia, Moller, Scheiner, \& Ryan, 2006; Rubin, Bukowski, \& Parker, 2006; Taylor, 2007). Friend support has traditionally focused on the quantitative features of the friendship network, such as size and density of the network, but the dyadic nature of the enacted friend support processes, especially among young adults, has rarely been examined (Carbery \& Buhrmester, 1998; Gillespie, Lever, Frederick, \& Royce, 2014). To this end, the overarching goal of the study is to unpack naturally occurring dynamic 
support seeking and provision behaviours within same-sex friendship dyads.

Social support in personal relationships is a dynamic process (Leatham \& Duck, 1990). Understanding this process requires considerations of individual person's experiences within a specific relationship dyad. Social support interactions (i.e., seeking and provision) occur in specific relationships; relationships are embedded in groups (e.g., gender groups or classes); groups dictate the features and patterns of the relationships and interactions that would likely arise in a given sociocultural context (Hinde, 1987; Rubin et al., 2006). As Hinde suggests, human experiences are inevitably influenced by different levels of social complexity. Conclusions drawn from a single level of analysis are limited in explaining the dynamic processes at work in human interactions. Guided by Hinde and Rubin et al., we aim to address both conceptual and analytical concerns in the investigation of social support processes by examining the three levels of social complexity (i.e., interaction, relationship, and group) and the macrosystem of cultural contexts impacting the three levels. We begin with a brief review of previous research on social support according to these three levels of social complexity.

\section{Interactions: Social Support Seeking and Provision}

Social support can be defined as, "the social resources that persons perceive to be available or that are actually provided to them by nonprofessionals in the context of both formal support groups and informal helping relationships" (Wills, 1991, p. 4). Two support seeking strategies have been identified: direct vs. indirect support seeking (Barbee et al., 1993; Barbee \& Cunningham, 1995). Direct support seeking includes overt and explicit behaviours containing necessary information to allow support transactions to occur, such as asking someone to pass the salt during dinner. Indirect support seeking includes behaviours that are more subtle and passive; it may vaguely signal the need for help, but without specifying what kind of help is needed. An example of indirect support seeking could be complaining to a friend about a difficult situation at work without asking for help directly. 
The vague and nonspecific behaviours of indirect support seeking make it more difficult for support providers to identify the actual needs of the support seekers. Eventually, indirect support seeking may even lead to a negative response because it is not clear for the support provider what specific help is needed (Barbee, 1990; Barbee \& Cunningham, 1995). Don et al. (2013) found evidence that for new parents, the use of indirect support seeking during pregnancy was related to elevated levels of perceived negative support responses at 1-month postpartum, which predicted indirect support seeking at 4-month postpartum, after controlling for relationship satisfaction. This study supported Barbee and Cunningham's argument that indirect support seeking may not be the best support seeking strategy due to its propensity to elicit negative responses from support providers.

Social support provided in dyads and small groups is often examined in three forms: informational, instrumental/tangible, and emotional/esteem (Cutrona \& Suhr, 1994; Taylor, 2007). Informational/tangible support provision, such as giving advice or lending money, centers around concrete problem-focused resolution of the stressor (Chen, Kim, Mojaverian, \& Morling, 2012; Chentsova-Dutton \& Vaughn, 2011). Emotional/esteem support provision, on the other hand, is an emotion-focused provision which aims at providing reassurance of the support recipient's positive self-worth, self-regard and emotional comfort (Taylor, 2007). Although social support usually refers to the positive aspects of social interactions, not all support responses would yield positive effects on the individual in need. For instance, people can provide poor advice (Shumaker \& Hill, 1991), be overly intrusive (Bolger, Foster, Vinokur, \& Ng, 1996) or simply engage in negative and counter-supportive behaviours (Crevier, Marchand, Nachar, \& Guay, 2014; Guay et al., 2011; Schuster et al., 1990), such as criticism, complains, refusal to help, disagreeing or arguing with the support seeker, interruption, and making hurtful or insensitive remarks of the situation (Cutrona \& Suhr, 1994; Don et al., 2013). In short, support provision comprises both positive (e.g., giving advice and encouragement) and negative (e.g., criticizing and being hurtful) aspects of social interactions. In the present research, we rely on problem-focused, emotion-focused and 
negative responses as the three facets of support provision among same-sex peers.

\section{Relationship: Friendship and Social Support}

Seeking for social support is a coping strategy that occurs primarily within the context of close relationships (e.g., Bokhorst, Sumter, \& Westenberg, 2010; Bolger et al., 2000; Bolger \& Amarel, 2007; Cable et al., 2013). Unlike parent-child or sibling relationships that are bonded by blood, friendship dyads are voluntary which make friendship particularly vulnerable because the loss of a single member will result in the termination of the relationship (Rubin et al., 2006). In this case, romantic relationships share some common ground with friendships in terms of voluntariness and membership size. However, previous research on the dyadic interactions of social support primarily focused on heterosexual married couples (e.g., Bolger et al., 2000; Bolger \& Amarel, 2007; Cutrona \& Suhr, 1994; Pasch, Bradbury, \& Davila, 1997; Sullivan, Pasch, Johnson, \& Bradbury, 2010) whereas research on social support amongst friendship dyads are scarce (Leatham \& Duck, 1990). In fact, friends are the second leading suppliers of support after spouses among married couples, and they are the single most frequent providers for support among single individuals (Carbery \& Buhrmester, 1998). As Muraco (2012) stated in her book: "friendship is one of the most significant, and yet socially ignored, relationships" (p. 15).

Starting from adolescence, friend support starts to exceed parental support for both boys and girls (e.g., Bokhorst et al., 2010; Day \& Livingstone, 2003). Previous research shows that adult men receive more familial support (Cable et al., 2013), whereas adult women benefit equally from friends and family (Derlega, Barbee, \& Winstead, 1994). High quality friendships include many positive features, such as prosocial behaviours, self-esteem support, intimacy, loyalty; negative features, such as criticism, conflicts, dominance attempts and rivalry, may also be present even in the best friendships (Berndt, 2002). In addition, supportive and negative interactions between intimate partners influence both the precipitating events and also the continuation, deterioration or dissolution of the intimate 
relationship (Cutrona, Russell, \& Gardner, 2005).

Social support is closely related to previous experiences in a specific relationship (Cutrona et al., 2005; Leatham \& Duck, 1990), and can also influence future interactions and the degree of relationship satisfaction (e.g., Lawrence et al., 2008). In the short term, how an individual asks for help from a friend has direct impact on the response he/she receives, and in turn influences how this individual asks for help at the next opportunity; in the long term, this particular social support interaction also has an impact on how the friend seeks help in the distant future due to the voluntary and reciprocal nature of friendship (Rubin et al., 2006). In sum, social support is both an interaction-specific and a relationship-specific phenomenon, which is also influenced by group and cultural contexts (Taylor, 2007).

\section{Group: Gender Effects}

As mentioned earlier, Don et al.'s (2013) study illustrated the reciprocal relation between indirect support seeking and perceived negative responses from new parents, but gender variations were neglected in their study. Barbee et al. (1993) argue that gender role expectations have significant influences on the differential support seeking behaviours between men and women. The female gender role, associated with nurturance and emotional expressiveness, places women in an advantageous situation in terms of openly soliciting help or receiving unsolicited help because women are expected to need help during times of distress. On the other hand, men who are expected to be competent and independent may be reluctant to seek help indirectly or directly because requesting for help or hinting help is needed makes them look weak and dependent (Barbee et al., 1993; Bolger \& Amarel, 2007). Consequently, the potential loss of face and threat to self-esteem may hinder men from seeking support. Cutrona et al. (2007), however, found that men and women were equally willing to seek and provide support. The gender variations in social support were actually due to the responsiveness of the support provider. For example, women were more 
responsive to their husbands during high distress days than were men to their wives (e.g., Neff \& Karney, 2005).

Barbee et al. (1993) also argue that men and women do not differ in the amount of help they provide but in the forms of help, with men more likely to provide instrumental help in an emergency situation which is congruent with male's gender role expectations, such as carrying a heavy package or fixing a door. Women, on the other hand, may provide more emotional-based support, such as comforting someone who just broke up with her boyfriend, which is also congruent with female's gender role expectations. However, other research showed that females were more likely to provide support in all forms, be it informational, tangible or emotional (Carbery \& Buhrmester, 1998). Gillespie et al. (2014) further attested that the gender differences in friendship may indeed be exaggerated, such that females would give advice and tangible support to their female friends in need and males would also share their feelings with their same-sex close friends. In other words, gender differences in emotional vs. instrumental support were in fact very small among same-sex friendships.

Furthermore, previous research showed some gender differences in adult friendships, such that women's best friendships were higher in quality, more supportive, more intimate, and lower in conflicts than men's (e.g., Bank \& Hansford, 2000; Demir \& Orthel, 2011). It is unclear whether the mixed results of gender differences observed in social support processes are influenced by the differential gender effects of friendship qualities among same-sex friendships. Therefore, the present research will continue to investigate the potential gender differences on social support processes by taking into account friendship qualities, both support seeking and provision behaviours simultaneously.

\section{Macrosystem: Cultural Variations}

Cultural variations based on different views of the self have great implications on social support processes (H. S. Kim \& Markus, 1999; H. S. Kim et al., 2008; Taylor et al., 2004; Wang, Shih, Hu, Louie, \& Lau, 2010). In individualistic cultural contexts, the self is 
understood as derived from its inner attributes, which are assumed to reflect the essence of the person (Markus \& Kitayama, 2010). People shaped by individualistic cultural contexts are then expected to take actions to express their opinions and realize their goals. Social relationships are assumed to be more autonomous with relatively few obligations. Hence, it is appropriate to actively seek support from others to cope with stress because social support is seen as a resource (H. S. Kim \& Markus, 1999; Taylor, 2011).

On the contrary, among collectivistic cultural contexts, such as those that predominate in Japan and China, the self can be viewed as a relational entity that is connected to and sustained by a number of significant relationships (e.g., family relations, occupational relations, and/or friendships). Here, the self is an extension of one's familial or social groups. The person, as a part of a group, is defined with reference to the larger whole (Markus \& Kitayama, 2010). Members of the collectivistic cultural contexts are expected to conform to social norms and seek consensus within the social group. In this case, personal goals and needs become secondary to maintaining social solidarity and harmony (H. S. Kim \& Markus, 1999). Consequently, it is inappropriate to burden others with one's own troubles because doing so might disrupt social harmony (H. S. Kim et al., 2008).

Direct support seeking may be perceived as troublesome because the need for support is a sign of incompetence and lack of self-efficacy in individualistic cultural contexts (Bolger \& Amarel, 2007); but it may also be perceived as problematic in collectivistic cultural contexts because imposing one's own problems on others might undermine social harmony (H. S. Kim et al., 2008; Park et al., 2013). In other words, cultural variations in social support seeking are based on self-oriented concerns for maintaining self-efficacy in individualistic cultural contexts, and other-oriented concerns for avoiding imposing troubles on others in collectivistic cultural contexts. For instance, Asians/Asian Americans are less likely to mobilize social support when coping with stressful negative and positive events (Taylor et al., 2004; Wang et al., 2010), compared to European Americans. Asians/Asian Americans may in fact prefer social support seeking in a more subtle manner, 
without disclosing their problems or stressful events explicitly (H. S. Kim et al., 2008). However, the studies that examined differential responses to direct vs. indirect support seeking behaviours were all conducted within "Western" cultural contexts (e.g., Barbee \& Cunningham, 1995; Don et al., 2013). Whether indirect support seeking would elicit negative responses among friendship dyads, as proposed by Barbee and colleagues, has not yet been examined in East Asian cultural contexts.

The benefits of social support are ubiquitous across many cultural contexts (Taylor, 2007), yet cultural variations not only exist in how support is sought, but also in how support is provided. In a questionnaire based study examining cultural differences of social support provision, Chen et al. (2012) showed that Euro-Americans provided more emotional/esteem support whereas Japanese participants provided more informational/tangible support in response to their close others' stressors. Chentsova-Dutton and Vaughn (2011) also found that unlike the Euro-Americans who preferred providing emotional support, Russians - who are also highly collectivistic - showed a strong preference for giving advice (i.e., informational support) regardless what type of support was sought. Furthermore, maintaining a harmonious relationship with others is a core concern in collectivistic cultural contexts (Markus \& Kitayama, 2010). Providing support to others is considered a social obligation in collectivistic cultural contexts, whereas it is dependent on the person's own volition in individualistic cultural contexts (H. S. Kim et al., 2008). The differential cultural meanings attached to relationship obligations might give rise to differential support transactions in different cultural contexts. Given the different stressors and sources of support providers (e.g., romantic partners or confederates) in previous studies examining support provision cross culturally, it is unclear whether these results from previous studies can be generalized to other collectivistic cultural contexts and whether these questionnaire based studies can represent the cultural variations in enacted support provision among same-sex friendships. Therefore, more studies are needed to investigate the preference for actual informational/tangible support provision among collectivistic cultural contexts. 


\section{The Current Study}

Interpersonal relationships, such as friendships, often create "nonindependence" between the two members of a dyad because people in close relationships influence each other's cognition, emotions, and behaviours (Kelley \& Thibaut, 1978; Kenny, Kashy, \& Cook, 2006; Rusbult \& Van Lange, 2008). Behavioural interactions in interpersonal relationships are dynamic and reciprocal. Current behavioural interactions are affected not only by the partner's reactions within the dyad but also by the relationship shared by the two participants in the interaction. This study therefore uses the Actor-Partner Interdependence Model (APIM; Kenny et al., 2006) to account for the nonindependence within friendships between the support seeker and support provider. Both actor and partner effects of APIM are examined simultaneously. An actor effect indicates how much a person's outcome variable is related to his/her own predictor, illustrated by the solid arrows in Figures 1 and 2; a partner effect indicates how much a person's outcome variable is associated with his/her partner's predictor, illustrated by the dash arrows. This dyadic design allows estimations of both intrapersonal effects (i.e., actor effects) and interpersonal effects (i.e., partner effects) in one model. There are four sets of questions we would like to investigate using the dyadic design of APIM.

\section{Research Question 1: Do positive features of friendship quality predict} more direct support seeking behaviours and supportive responses? The perception of relationship quality is largely based on previous experiences shared between the two friends (e.g., Cutrona et al., 2005) and this perception of relationship can influence future interactions in the dyad (e.g., Leatham \& Duck, 1990). Even though it has been repeatedly argued that the association between relationships and actual interactions are dynamically interlocked, relationship satisfaction or relationship qualities have often been measured as the outcome of social support processes - but not the other way around among friendship dyads. A few studies on romantic relationships suggest that perceived positive relationship quality is indeed positively related to perceived support and observed 
supportive behaviours (e.g., Don et al., 2013; Gurung, Sarason, \& Sarason, 1997). Following the same line of logic, we hypothesized that positive friendship quality was expected to associate with higher levels of supportive responses (i.e., problem-focused and emotion-focused support; actor effect for the support provider). Similarly, since positive friendship could create a safe haven for friends to disclose their problems or concerns directly, we also hypothesized that positive friendship quality would be associated with higher levels of direct support seeking behaviours (actor effect for the support seeker). Direct support seeking would be positively related to supportive responses after controlling for both actor and partner effects of positive friendship quality.

Partner effects are the interpersonal effects that signify the mutual influences between the two members of the dyad (Kenny et al., 2006). Understanding the interpersonal aspects of social support is as important as understanding the intrapersonal effects (Rusbult \& Van Lange, 2008). However, as the partner and actor effects have not yet been studied simultaneously in relation to social support processes among friends, we consider the partner effects to be exploratory in this study and no specific hypothesis for partner effects were posited. Nevertheless, considering social support transactions in intimate relationships are reciprocal (e.g., Cutrona et al., 2005) and partners in close relationships can influence each others' emotions and behaviours (Kenny et al., 2006; Kenny \& Kashy, 2011; Rubin et al., 2006; Verhofstadt et al., 2012), the partner effects are presumed to show similar directions and patterns of influences as actor effects in this study.

\section{Research Question 2: Do negative features of friendship quality predict} more indirect support seeking behaviours and negative responses? Previous negative interactions within the dyad might inhibit support seekers to reveal their needs directly because they are afraid their requests would be rejected or criticized (Barbee et al., 1993; Barbee \& Cunningham, 1995; Don et al., 2013). Given negative perception on friendship may hinder friends to seek help directly, perceived negative friendship quality was expected to predict higher levels of indirect support seeking behaviours (actor effect for the 
help seeker), and it would also positively relate to negative responses (actor effect for the help provider). Indirect support seeking would be positively related to negative responses after controlling for both actor and partner effects of negative friendship quality.

\section{Research Question 3: Are there gender variations in support seeking and}

provision behaviours? Since gender role expectations are broadly similar in both "Western" and "Asian" cultural contexts (Chueng, 1996; Granrose, 2006), gender variations in social support seeking and provision were expected to be similar between the two cultural contexts. Therefore, we hypothesized that female participants would seek more help than male participants. Following previous research (Barbee et al., 1993; Carbery \& Buhrmester, 1998), female participants were expected to provide more support (i.e., both problem-focused and emotion-focused) than male participants controlling for friendship quality. Furthermore, we expected that the interaction between friendship quality and gender would be significant, with the effects of friendship quality on social support seeking and provision would be stronger among female participants than male participants.

\section{Research Question 4: Are there cultural variations in support seeking and} provision behaviours? In line with H. S. Kim et al. (2008), we expected that Chinese participants in our study would use more indirect support seeking than would Euro-Canadian participants. Since support provision is considered an obligation in collectivistic cultural contexts (H. S. Kim et al., 2008; Park et al., 2013; Taylor, 2007), Chinese participants were expected to be more inclined to provide support to their friends than Euro-Canadians. According to Chen et al. (2012) and Chentsova-Dutton and Vaughn (2011), it was also expected that Chinese participants would provide more problem-focused support, whereas Euro-Canadians would provide more emotion-focused support after friendship quality was taken into account. Furthermore, since group harmony is a major concern in collectivistic cultural contexts (H. S. Kim et al., 2008; Markus \& Kitayama, 2010), overt negative behaviours that disrupt group harmony might be less frequent during social interactions within close relationships in collectivistic cultural contexts. We hypothesized 
that Chinese participants might be less likely to use negative behaviours as a response in comparison to Euro-Canadians. We also expected that the interaction between friendship quality and cultural contexts would be significant, with the effects of friendship quality would be stronger for Chinese participants than for Euro-Canadian participants.

\section{Method}

Participants. Participants were recruited through advertisements posted on a departmental participant pool website, local student association websites and throughout campus in an English-speaking university in Montréal. Participants who contacted us were asked to bring a same-sex non-romantic friend of a similar cultural background to participate in this study with them. Each participating dyad in this study consisted of two members of the same-sex and of the same cultural background. Euro-Canadian dyads (26 female dyads, mean age $=20.42 ; 10$ male dyads, mean age $=24.25)$ were all born in Canada and self-identified as (a) having European heritage; (b) being "White (Caucasian)". Chinese dyads (32 female dyads, mean age $=24.92 ; 24$ male dyads, mean age $=24.79)$ were all born in China and left China after the age of eight. The Chinese sample in this study consisted of either registered full-time international students (79.5\%) or immigrants (20.5\%).

We refer to one of the participants (randomly assigned) in each dyad as the Target and his/her friend as the Friend. Most of our participants (53.3\%) were single, $34.2 \%$ in a relationship, 9.8\% married or cohabiting, and 2.7\% separated or divorced. Nobody considered their partner in the study as acquaintances or colleagues. The majority of the participants (79.3\%) regarded their partner in the dyad as their best or good friend, and $20.7 \%$ regarded their counterpart in the dyad as their friend.

Procedures. Participants completed online questionnaires related to demographic information (e.g., gender, age, cultural background and language proficiency) and a questionnaire regarding friendship quality with their friend prior to the laboratory visit. The gender of the experimenter was matched with the gender of the participants in each lab visit. 
Participants came with their friends in pairs and they were first left alone inside the testing room for five minutes to habituate to the lab environment. The experimenter then returned and instructed one of the participants (the Target) to copy a picture depicting downtown Boston using an Etch-A-Sketch board in 10 minutes. The Friend was instructed to help if he/she wanted, but also instructed that he/she could not do the task for the Target. Since the purpose of this study was to investigate how friends provide and ask for help during distressing situations, this Etch-A-Sketch task presents a challenge that mimics a non-social daily hassle (e.g., preparing for a final exam) but it is not as threatening to the self-esteem as solving a set of math problems (Mojaverian \& Kim, 2013) nor is it as self-disclosing as talking about personal feelings. Therefore, this task presents a low cost scenario to the Target and the Friend, allowing support seeking and provision to occur naturally without posing a serious threat to self-esteem and self-efficacy. The choice of a challenging but non-math task strengthens the validity of the present study. After this Etch-A-Sketch task, participants completed several other filler tasks, such as completing additional questionnaires that were not relevant to the current study ${ }^{1}$.

The experimenter answered any questions before starting the task. At the end of the lab visit, the experimenter thanked both participants and provided debriefing forms. All participants chose from one of three compensation methods: 1) four participant pool credits; 2) two participant pool credits with $\$ 20$ cash; 3) $\$ 20$ cash if not eligible for participant pool credits. Participants provided their written consent to participate prior to filling out the questionnaires and attending the lab visit. They were informed they could withdraw from the study anytime they wish. The study protocol and informed consent were approved by the university's ethics review board.

\footnotetext{
${ }^{1}$ The original study also included a Webcam Task, which always followed the Etch-A-Sketch Task. Results from the Webcam Task followed a largely, although not entirely, similar pattern to those from the Etch-ASketch Task. Due to the length of the full manuscript originally submitted, the peer reviewers suggested dropping the Webcam Task from the final report. Results from the Webcam Task, and from cross-task analyses, are available upon request.
} 
Measures. The Network of Relationships Inventory - Behavioural System Version (NRI; Furman \& Buhrmester, 2009) were administered to each participant separately regarding the quality of their relationship with their friend in the study. The NRI consists of 24 items that assess both positive and negative features of social interactions in different relationships using a five-point Likert-type scale (ranging from $1=$ "Little or None" to $5=$ "The Most"). The positive features of relationships, including attachment, caregiving and affiliation, are assessed in the "Supportive Interaction Scale", whereas the negative features of relationships, including conflict, antagonism and criticism, are measured in the "Negative Interaction Scale". A sample item for the "Supportive Interaction Scale" is: "How much do you seek out this person when you are upset?". A sample item for the "Negative Interaction Scale" is: "How much do you and this person say mean or harsh things to each other?" Participants filled out this questionnaire online prior to the lab visit. In the subsequent analyses, we will refer to the "Supportive Interaction Scale" as the supportive NRI, and the "Negative Interaction Scale" as the negative NRI.

Seven participants provided incomplete data on the NRI. Little's Missing Completely At Random (MCAR) tests using SPSS v22 were conducted for the Targets and the Friends separately to avoid statistical interdependence between participants. Results showed that responses were missing completely at random, $\chi^{2}(14)=12.59, p=.56$ for the Targets and $\chi^{2}(23)=15.28, p=.88$ for the Friends in the supportive NRI ( 15 items). The negative NRI (9 items) was also missing completely at random for the Friends, $\chi^{2}(7)=11.78, p=.11$. There were no missing items for Targets in the negative NRI. Therefore, Maximum-Likelihood data imputations were employed for Targets and Friends separately in each scale using the Expectation-Maximization algorithm (McKnight, McKnight, Sidani, \& José, 2007). Mean scores for each scale were then calculated. Cronbach's alpha coefficients for supportive NRI were $\alpha=.97$ for Euro-Canadians and $\alpha=.96$ for Chinese. Cronbach's alpha coefficients for the negative NRI were $\alpha=.81$ for Euro-Canadians and $\alpha=.85$ for Chinese. 
Language Proficiency consists of three language related questions. Chinese participants were asked to rate their level of proficiency in speaking, understanding, and writing in English using a 7 -point rating scale with $1=$ "Very poor" to $7=$ "Native-like". The mean level of English proficiency was 4.68, $S D=0.96$. Students who were attending English as a Second Language classes were not recruited for this study. The recruitment criteria, verified by the language proficiency questions, indicated that Chinese participants in this study have adequate English proficiency to understand the online questionnaires and instructions during the lab visits.

The frequency of social support seeking behaviours was coded using the combination of the Social Support Elicitation Behaviour Code (SSEBC) developed by Cutrona, Suhr, and MacFarlane (1990) which contains verbal support seeking, and the nonverbal support seeking behaviours conceptualized by M. S. Kim, Shin, and Cai (1998). This study used event sampling method to code two types of support seeking behaviours. Indirect support seeking (IDSS) was coded when the Target exhibited difficulty in performing the task but did not indicate directly what kind of help was needed. Examples of IDSS include: "this is so difficult" or "Jesus!" Nonverbal example of IDSS includes: the Target stops the task and looks at the Friend with prolonged silence, sighing or sulking. The code of direct support seeking (DSS) was assigned if the behaviour explicitly showed support was needed. For instance: "How can I draw a tree?" The inter-rater reliability on $15 \%$ of the cases was Kappa $=.91$.

The frequency of social support provision behaviours was coded using the Social Support Behaviour Code (SSBC Suhr, Cutrona, Krebs, \& Jensen, 2004). The coding manual includes three supportive codes: information support (e.g., "You can draw the building first."), emotional and esteem support (e.g., "You are doing great!") and tangible support (e.g., holding the jiggling Etch-A-Sketch board). Informational and tangible support were grouped together to create the problem-focused support and the emotional and esteem support were grouped together to create the emotion-focused support (Chen et al., 2012). 
Negative behaviours (NB) were coded as comments or behaviours that would jeopardize or discourage the completion of the task by criticizing or complaining. An example would be "That looks terrible". The inter-rater reliability on $15 \%$ of the cases was Kappa $=.84$.

\section{Results}

\section{Preliminary Analysis}

Table 1 presents the means and standard deviations, and Table 2 presents the correlations of all variables for Euro-Canadian and Chinese participants. In order to obtain a general picture of cultural and gender variations on social support processes, we first used cultural contexts and gender as independent variables to predict support seeking and provision behaviours separately without considering friendship qualities. Because the dependent variables (i.e., support seeking and provision behaviours) were frequency count data, and distribution fit analysis (Sheppard, 2012) using MATLAB R2012a showed that all coded variables of interest fitted best to negative binomial distributions, simple negative binomial regression with a Log link function was used in the preliminary analyses in SPSS v22 (see Table 3).

Without taking friendship qualities into account, Euro-Canadian Targets used indirect support seeking about two times more than Chinese Targets, but they were about $35 \%$ less likely than Chinese Targets to use direct support seeking. For support provision, Euro-Canadian participants were $36 \%$ less likely to provide problem-focused support to their friends than Chinese participants, but Euro-Canadians were $72 \%$ more likely to provide emotion-focused support than Chinese participants. Furthermore, Euro-Canadians were almost 3 times more likely than Chinese to display negative behaviors during the experimental task.

There were also some gender variations in the preliminary analyses. Males were less likely to seek help than females in general, with $31 \%$ less likely for indirect support seeking and $43 \%$ less likely for direct support seeking. Males were also less likely to provide 
problem-focused support (22\% less), emotion-focused support (24\% less) and display negative behaviours (46\% less) than females according to our analyses. In sum, males sought less and provided less support to their friends during the experimental task.

Insert [Table 1, Table 2 and Table 3] here.

\section{APIM Analysis}

The preliminary analyses showed that there were both cultural and gender variations in social support seeking and provision behaviours. Since social support interactions are not isolated behaviours but rather occur within close relationships in a dynamic manner, we will be examining whether similar patterns of cultural and gender variations continue to exist when we take friendship qualities into consideration. The Generalized Estimating Equation (GEE) with Log link function was used to estimate the parameters of APIM (Loeys \& Molenberghs, 2013). The two-intercept approach was used to create two separate equations with two intercepts: one for the Target and one for the Friend (see Appendix for equations; Kenny et al., 2006; Loeys \& Molenberghs, 2013). By doing so, the results can be directly interpreted according to participant's roles as the Target or the Friend, accounting for both interpersonal and intrapersonal effects in the same model (Kenny \& Kashy, 2011; Wickham \& Knee, 2012).

Two sets of APIMs were conducted. Model 1: Cultural group (Chinese $=1$, Euro-Canadians $=-1$ ) was entered into the Standard APIM as a moderator; Model 2: Gender $($ female $=1$, male $=-1$ ) was entered also as a moderator. Because we used the two-intercept approach to conduct our APIM analysis, by entering a moderator into the models, it would create a three-way interaction in our APIM analyses Target(orFriend) $* N R I *$ Culture for Model 1 and Target(orFriend) $* N R I *$ Gender for

Model 2. Both supportive and negative NRI variables were grand mean centered prior to the APIM analysis. Marginal $R^{2}$, which reveals the proportion of variance explained by the fitted model (Hardin \& Hilbe, 2003), are presented in all APIM effect tables. All APIM 
related analyses were conducted using the "PROC GENMOD" command in SAS v9.3 following the procedures specified in Loeys, Cook, De Smet, Wietzker, and Buysse (2014).

\section{Research Questions 1, 3 \& 4: direct support seeking and problem-focused support}

In this set of analyses (see Figure 1), Target's partner main effect positively predicted problem-focused support (see Table 4). Every one unit increase in Target's supportive NRI was related to $33.1 \%^{2}$ more problem-focused support provided by the Friend (Target's partner main effect). No significant three-way interaction was obtained using APIM with cultural context as a moderator (Model 1). There were no significant main effects or gender variations for predicting direct support seeking and problem-focused support in Model 2. The partial correlations between direct support seeking and problem-focused support were $.69^{3}$ in Model 1 and .80 in Model 2.

Insert [Table 4 and Figure 1] here.

\section{Research Questions 1, 3 \& 4: direct support seeking and emotion-focused support}

The same sets of APIM models were analyzed using direct support seeking and emotion-focused support as outcome variables (see Figure 2). Results in Model 1 using cultural context as the moderator showed that both Target's and Friend's main effects of supportive NRI were related to emotion-focused support provided by the Friend (Table 4). Higher supportive NRI perceived by the Target was associated with more emotion-focused support provided at a rate of $33.9 \%$ (Target's partner main effect), and higher supportive NRI perceived by the Friend was associated with more emotion-focused support provided at

\footnotetext{
${ }^{2}$ The exponentiated estimates (Rate Ratios) indicate the amount of change in the dependent variable for every unit increase in the independent variable, controlling for all other effects in the model (Loeys et al., 2014).

${ }^{3}$ All partial correlations have accounted for both actor and partner effects. The partial correlation obtained using the "unstructured working correlation" in the GEE procedure does not come with significance test or standard error because it is a nuisance parameter (Loeys \& Molenberghs, 2013).
} 
a rate of $21.5 \%$ (Friend's actor main effect). No significant three-way interaction was obtained using cultural context as a moderator. The partial correlation between direct support seeking and emotion-focused support in Model 1 was .69.

Results in Model 2 using gender as the moderator showed that there were no significant main effects. However, there was a significant three-way interaction for Friend's actor effect - higher supportive NRI perceived by the Friend was related to more emotion-focused support provided by female friends at a rate of $32.6 \%$. On the contrary, higher supportive NRI perceived by the Friend was related to less emotion-focused support provided by males at a rate of $12.7 \%$. The partial correlation between direct support seeking and emotion-focused support in Model 2 was .44.

Insert [Figure 2 and Figure 3] here.

\section{Research Questions 2, 3 \& 4: indirect support seeking and negative responses}

Regarding the analyses of indirect support seeking and negative behaviours (Figure 4), no significant results were obtained for Model 1 (analysis for cultural variations; see Table 5). Target's actor and partner main effects were significant in Model 2 (analysis for gender variations). The significant main effects showed that with one unit increase in the Target's negative NRI, there was about $30.3 \%$ decrease in the use of indirect support seeking by Target participants (Target's actor main effect), and there was a $32.5 \%$ decrease of negative behaviours provided by the Friend (Target's partner main effect). Moreover, there were no significant three-way interactions for either cultural or gender variations. The partial correlations between indirect support seeking and negative responses were .13 in Model 1 and .21 in Model 2.

Insert [Table 5 and Figure 4] here.

\section{Discussion}

Social support plays an important role in individual's well-being and the development of intimate relationships (Cutrona et al., 2005; Park et al., 2013; Taylor, 2007). Our 
experimental study used Actor-Partner Interdependence Models to incorporate cultural and gender influences into our understanding of social support seeking and provision within same-sex friendships. The GEE-approach used for analyzing the APIMs (Loeys \& Molenberghs, 2013) provided appropriate estimates for the frequency count outcome variables of support transactions between the Friend and the Target. Our goal in this study was to identify not only intrapersonal but also interpersonal effects on enacted social support seeking and provision behaviours, taking friendship qualities, cultural and gender variations into consideration. Consistent with our hypotheses and previous research (Barbee et al., 1993; Barbee \& Cunningham, 1995), direct support seeking was indeed positively related to both problem-focused and emotion-focused support after controlling for actor and partner

effects of perceived supportive friendships among same-sex friends. Indirect support seeking was also found to be positively related to negative behaviours after controlling for actor and partner effects of perceived negative friendships.

Although there were no significant three-way interactions on partner effects in this study, partner main effects were apparent in our analyses, indicating the necessity of including both actor and partner effects while examining social support processes. Findings in partner main effects further supported interdependence theory that dyadic members in a relationship have mutual influences on each other's emotion, cognition and behaviours (Kenny et al., 2006; Keppel \& Wickens, 2004; Rusbult \& Van Lange, 2008; Sadler, Ethier, \& Woody, 2011; Wickham \& Knee, 2012). The examination of either actor or partner effects in separate models is inadequate while studying social interactions in dyadic relationships.

\section{Cultural Variations}

In the preliminary analyses, simple negative binomial regression analyses using cultural context as a predictor revealed that Euro-Canadian participants were less likely to use direct support seeking and more likely to use indirect support seeking in the Etch-A-Sketch task than Chinese Target participants. These findings are different from previous research (e.g., 
H. S. Kim, Sherman, Ko, \& Taylor, 2006; H. S. Kim et al., 2008; Mojaverian \& Kim, 2013). However, Chinese participants were more likely to provide problem-focused support but Euro-Canadian participants were more likely to provide emotion-focused support, which are consistent with previous research (Chen et al., 2012; Chentsova-Dutton \& Vaughn, 2011). In our experimental task, the support seekers and the providers were informed the purpose of the tasks together. It is likely that Chinese participants no longer hesitated to ask for help directly because the Friend's role to help had been clearly defined and the need for help was justified in the instruction (Park et al., 2013). The Friend's help therefore is expected, because helping when needed is considered a social obligation and it is very central to the definition of friendship in Chinese cultural contexts (Adams \& Plaut, 2003; H. S. Kim et al., 2008; Gummerum \& Keller, 2008). Therefore, Chinese participants were as likely as Euro-Canadian participants to ask for help directly or indirectly. In addition, most of the experimental studies on culture and social support were not examined in friendship dyads nor on actual support seeking behaviours. The use of a confederate (e.g., Mojaverian \& Kim, 2013), who had no prior relation with the participants, may have inhibited participants from asking for help because no social obligation is attached to a stranger to offer help in both Chinese and Euro-Canadian cultural contexts. Moreover, support seeking behaviours were often examined using self-report measures without any considerations of actual supportive or negative responses during the social support interactions (e.g., H. S. Kim et al., 2006). Consequently, previous studies have only revealed cultural variations on the perception of social support seeking across relationships, but not actual support seeking behaviours in specific relationships as in our study presented here.

Our study used APIM to examine both the help seeker's and the helper's interpersonal (i.e., partner effects) and intrapersonal (i.e., actor effects) effects simultaneously, whereas previous studies often examined actor and partner effects in separate models with some exceptions (e.g., Crocker \& Canevello, 2008; Cutrona et al., 2007; Sullivan et al., 2010; Wang \& Repetti, 2014). Our analyses reflected the dynamic nature of social support processes in 
different levels of social complexity instead of only focusing on one level of analysis. When only investigating cultural variations on support seeking and provision separately without considering other levels of social complexity, such as relationship and actual interactions, we found evidence of cultural variations on both support seeking and provision behaviours in the preliminary analyses. However, once relationship quality, actual social support interactions and the macrosystem of cultural contexts were considered together in one model (i.e., APIM), the cultural variations no longer exist. Since social support often occurs within intimate relationships (Leatham \& Duck, 1990), the consideration of the relationship and actual interactions occurring in that particular relationship are imperative for a thorough understanding of social support processes. Our findings supported Hinde (1987) that the different levels of social complexity are all interlocked together and they must be examined together because the investigation of only one level of social complexity will produce biased results otherwise (Rubin et al., 2006; Rusbult \& Van Lange, 2008).

\section{Gender Variations}

Simple negative binomial regression analyses showed that male participants were less likely than female participants to seek or provide support. However, the significant three-way interactions indicated that supportive friendships were associated with more provision of emotion-focused support, with female participants providing higher rates of emotion-focused support during the Etch-A-Sketch task than male participants. This finding is consistent with the gender role expectation theory (Barbee et al., 1993). Females are more expressive in

their emotions (Denton, Prus, \& Walters, 2004; Wilhelm, 2014) and their friendship networks are often more intimate than males, therefore, it is no surprise that they are more likely to show compassion and display emotional support when their same-sex friends are in distress.

Furthermore, the lack of gender variations in support seeking behaviours in APIM analyses may be explained by the supportive nature of friendships. Supportive friendships could foster a positive and safe environment for social interactions to occur (Cutrona et al., 
2005), and the potential risk of undermining one's competence when asking for help is minimized. As a result, support seekers are willing to ask for help directly. On the one hand, Barbee et al. (1993) proposed that men's diminished use of support seeking is due to their fear of a potential threat to their self-esteem. On the other hand, our findings suggest that once this threat to the self is removed or minimized, such as being in a supportive relationship, there are no gender differences on support seeking. Males are equally likely to seek help as females as long as they perceive the relationship as safe and supportive.

Similar to the findings of cultural variations, the effects for gender variations disappeared after taking different levels of social complexity into account, except for emotion-focused support. The discrepancies in findings between one single level of analysis (i.e., the negative binomial regressions) and multiple levels of analyses (i.e., the analyses using APIM) highlight the elusiveness of reflecting individual's experiences by examining behaviours within one level of analysis without considering in what relationship these behaviours occur, and under what circumstances or influences these behaviours are most prominent. Our approach to bridging the theoretical model of multiple levels of social complexity and the analytical model of APIM provided a more comprehensive understanding of cultural and gender variations on enacted social support processes among same-sex friendship dyads.

\section{Limitations, Future Directions and Implications}

The present study has some limitations that should be taken into consideration while interpreting the results. Social interactions among friends consist of both positive and negative interactions. Positive interactions between two friends may influence future negative interactions and vice versa (e.g., Burleson, 1990; Rubin et al., 2006). The investigation of the relationship between positive and negative interactions among friends is beyond the scope of this study, and Furman and Buhrmester (2009) argued that these are two distinctive aspects of peer interactions which should be examined separately. Nonetheless, future studies might 
consider a longitudinal approach to examine the mutual influences between positive and negative interactions.

Due to the complexities of the models we analyzed, it was not possible to examine the interaction between cultural group and gender. Nor can we run separate analysis for Chinese vs. Euro-Canadian participants because the sample size for each cultural context is too small. Furthermore, we had fewer male dyads than female dyads in our data (i.e., 34 male dyads vs. 58 female dyads). Future studies should replicate our findings with similar numbers of male vs. female dyads and analyze them within different cultural contexts. Since most of our participants are university students, replication with non-student populations is necessary to test the generalizability of the results obtained here.

Cultural contexts shape people's understanding and perception of relationships, which in turn influence their responses to support seeking and provision (Jacobson, 1987; Taylor et al., 2004). Future cultural/cross-cultural studies of social support should employ both self-reports and observed behavioural measures to investigate how and when perceived support is being translated into actual supportive/negative responses in close relationships. This study focused on dyads that have the same sex and same cultural backgrounds. Given the ethnocultural diversity of modern industrial societies, where intergroup interactions among same-sex and cross-sex friends are frequent (Muraco, 2012), it will prove fruitful to extend this line of research to intergroup relationships and other cultural contexts to further unpack the cultural influences on social support interactions.

Despite these limitations, the Actor-Partner Interdependence Model used in this study preserved the partner influences on social support, which was often lost in studies with a sole focus on actor effects. Our study also demonstrated the importance and feasibility of investigating multiple levels of social complexity (i.e., interaction, relationship, group and cultural contexts) simultaneously with an APIM approach. Besides of the methodological and analytical implications, this study could also inform help seeking behaviours in the healthcare system. It has been well documented in epidemiology and social sciences studies 
that men are more reluctant than women to seek help actively when encountering health or mental health issues (e.g., Galdas, Cheater, \& Marshall, 2005; Kessler, Brown, \& Broman, 1981; Tamres, Janicki, \& Helgeson, 2002). Our analysis of gender effects suggest that these gender differences may be exaggerated if help seeking occurs within a supportive relationship. Health services or institutes perhaps should create a supportive environment for patients and promote judgement-free assessments for illnesses, particularly in the context of psychological counseling or psychiatric services in order to avoid delayed diagnosis or delayed treatment for men. 


\section{References}

Adams, G., \& Plaut, V. C. (2003). The cultural grounding of personal relationship: Friendship in North American and West African worlds. Personal Relationships, 10(3), 333-347. doi: 10.1111/1475-6811.00053

Bank, B. J., \& Hansford, S. L. (2000). Gender and friendship: Why are men's best same-sex friendships less intimate and supportive? Personal Relationships, 7(1), 63-78. doi: 10.1111/j.1475-6811.2000.tb00004.x

Barbee, A. P. (1990). Interactive coping: the cheering-up process in close relationships. In S. Duck \& R. Cohen (Eds.), Personal relationships and social support (p. 46-47). London ; Newbury Park : Sage Publications.

Barbee, A. P., \& Cunningham, M. R. (1995). An experimental approach to social support communications: Interactive coping in close relationships. Communication Yearbook, 18, 381-413.

Barbee, A. P., Cunningham, M. R., Winstead, B. A., Derlega, V. J., Gulley, M. R., Yankeelov, P. A., \& Druen, P. B. (1993). Effects of gender role expectations on the social support process. Journal of Social Issues, 49(3), 175-190. doi: 10.1111/j.1540-4560.1993.tb01175.x

Berndt, T. J. (2002). Friendship quality and social development. Current Directions in Psychological Science, 11(1), 7-10. doi: 10.1111/1467-8721.00157

Bokhorst, C. L., Sumter, S. R., \& Westenberg, P. M. (2010). Social support from parents, friends, classmates, and teachers in children and adolescents aged 9 to 18 years: Who is perceived as most supportive? Social Development, 19(2), 417-426. doi: $10.1111 / \mathrm{j} .1467-9507.2009 .00540 . \mathrm{x}$

Bolger, N., \& Amarel, D. (2007). Effects of social support visibility on adjustment to stress: Experimental evidence. Journal of Personality and Social Psychology, 92(3), 458-475. doi: $10.1037 / 0022-3514.92 .3 .458$

Bolger, N., Foster, M., Vinokur, A. D., \& Ng, R. (1996). Close relationships and 
adjustments to a life crisis: The case of breast cancer. Journal of Personality and Social Psychology, 70(2), 283-294. doi: 10.1037/0022-3514.70.2.283

Bolger, N., Zuckerman, A., \& Kessler, R. C. (2000). Invisible support and adjustment to stress. Journal of Personality and Social Psychology, 79(6), 953-961. doi: 10.1037/0022-3514.79.6.953

Burleson, B. R. (1990). Comforting as social support: relational consequences of supportive behaviors. In S. Duck \& R. Cohen (Eds.), Personal relationships and social support (p. 66-82). London ; Newbury Park : Sage Publications.

Cable, N., Bartley, M., Chandola, T., \& Sacker, A. (2013). Friends are equally important to men and women, but family matters more for men's well-being. Journal of Epidemiology and Community Health, 67(2), 166-171. doi: 10.1136/jech-2012-201113

Carbery, J., \& Buhrmester, D. (1998). Friendship and need fulfillment during three phases of young adulthood. Journal of Social and Personal Relationships, 15(3), 393-409. doi: $10.1177 / 0265407598153005$

Chen, J. M., Kim, H. S., Mojaverian, T., \& Morling, B. (2012). Culture and social support provision: Who gives what and why. Personality and Social Psychology Bulletin, 38(1), 3-13. doi: $10.1177 / 0146167211427309$

Chentsova-Dutton, Y. E., \& Vaughn, A. (2011). Let me tell you what to do: Cultural differences in advice-giving. Journal of Cross-Cultural Psychology, 43(5), 687-703. doi: $10.1177 / 0022022111402343$

Chueng, F. M. (1996). Gender role development. In Growing up the Chinese way: Chinese child and adolescent development. The Chinese Univeristy of Hong Kong.

Crevier, M. G., Marchand, A., Nachar, N., \& Guay, S. (2014). Overt social support behaviors: Associations with ptsd, concurrent depressive symptoms and gender. Psychological Trauma: Theory, Research, Practice, and Policy, 6(5), 519-526. doi: $10.1037 / \mathrm{a} 0033193$

Crocker, J., \& Canevello, A. (2008). Creating and undermining social support in communal 
relationships: The role of compassionate and self-image goals. Journal of Personality and Social Psychology, 95(3), 555-575. doi: 10.1037/0022-3514.95.3.555

Cutrona, C. E., Russell, D. W., \& Gardner, K. A. (2005). The relationship enhancement model of social support. In T. A. Revenson, K. Kayser, \& G. Bodenmann (Eds.), Couples coping with stress: Emerging perspectives on dyadic coping. (p. 73-95). American Psychological Association. doi: 10.1037/11031-000

Cutrona, C. E., Shaffer, P. A., Wesner, K. A., \& Gardner, K. A. (2007). Optimally matching support and perceived spousal sensitivity. Journal of Family Psychology, 21(4), 754-758. doi: 10.1037/0893-3200.21.4.754

Cutrona, C. E., \& Suhr, J. A. (1994). Social support communication in the context of marriage: An analysis of couples' supportive interactions. In B. R. Burleson, T. L. Albrecht, \& I. G. Sarason (Eds.), Communication of social support: Messages, interactions, relationships, and community (p. 113-135). Thousand Oaks, CA, US: Sage Publications, Inc.

Cutrona, C. E., Suhr, J. A., \& MacFarlane, R. (1990). Interpersonal transcations and the psychological sense of support. In S. Duck \& R. Cohen (Eds.), Personal relationships and social support (p. 30-45). London: Sage Publications, Inc.

Day, A. L., \& Livingstone, H. A. (2003). Gender differences in perceptions of stressors and utilization of social support among university students. Canadian Journal of Behavioural Science/Revue canadienne des sciences du comportement, 35(2), 73-83. doi: $10.1037 / \mathrm{h} 0087190$

Deci, E. L., La Guardia, J. G., Moller, A. C., Scheiner, M. J., \& Ryan, R. M. (2006). On the benefits of giving as well as receiving autonomy support: Mutuality in close friendships. Personality and Social Psychology Bulletin, 32(3), 313-327. doi: $10.1177 / 0146167205282148$

Demir, M., \& Orthel, H. (2011). Friendship, real-ideal discrepancies, and well-being: Gender differences in college students. The Journal of Psychology, 145(3), 173-193. doi: 
$10.1080 / 00223980.2010 .548413$

Denton, M., Prus, S., \& Walters, V. (2004). Gender differences in health: a canadian study of the psychosocial, structural and behavioural determinants of health. Social Science E6 Medicine, 58(12), 2585-2600. doi: 10.1016/j.socscimed.2003.09.008

Derlega, V. J., Barbee, A. P., \& Winstead, B. A. (1994). Friendship, gender, and social support: Laboratory studies of supportive interventions. In G. R. Burleson, T. L. Albrecht, \& I. G. Sarason (Eds.), Communication of social support: messages, interactions, relationships, and community (p. 136-151). Thousand Oaks, CA, US: Sage Publications, Inc.

Don, B. P., Mickelson, K. D., \& Barbee, A. P. (2013). Indirect support seeking and perceptions of spousal support: An examination of a reciprocal relationship. Personal Relationships, 20(4), 655-668. doi: 10.1111/pere.12006

Furman, W., \& Buhrmester, D. (2009). The network of relationships inventory: Behavioral systems version. International Journal of Behavioral Development, 33(5), 470-478. doi: $10.1177 / 0165025409342634$

Galdas, P. M., Cheater, F., \& Marshall, P. (2005). Men and health help-seeking behaviour: literature review. Journal of Advanced Nursing, 49(6), 616-623. doi: 10.1111/j.1365-2648.2004.03331.x

Gillespie, B. J., Lever, J., Frederick, D., \& Royce, T. (2014). Close adult friendships, gender, and the life cycle. Journal of Social and Personal Relationships, 32(6), 709-736. doi: $10.1177 / 0265407514546977$

Goldsmith, D. J. (2004). Communicating social support. Cambridge University Press.

Granrose, C. S. (Ed.). (2006). Employment of women in Chinese cultures: Half the sky. Edward Elgar Publishing, Inc.

Guay, S., Beaulieu-Prévost, D., Beaudoin, C., St-Jean-Trudel, E., Nachar, N., Marchand, A., \& O'Connor, K. P. (2011). How do social interactions with a significant other affect PTSD symptoms? An empirical investigation with a clinical sample. Journal of 
Aggression, Maltreatment \&3 Trauma, 20(3), 280-303. doi: $10.1080 / 10926771.2011 .562478$

Gummerum, M., \& Keller, M. (2008). Affection, virtue, pleasure, and profit: Developing an understanding of friendship closeness and intimacy in Western and Asian societies. International Journal of Behavioral Development, 32(3), 218-231. doi: $10.1177 / 0165025408089271$

Gurung, R. A. R., Sarason, B. R., \& Sarason, I. G. (1997). Personal characteristics, relationship quality, and social support perceptions and behavior in young adult romantic relationships. Personal Relationships, 4(4), 319-339. doi:

10.1111/j.1475-6811.1997.tb00149.x

Hardin, J., \& Hilbe, J. (2003). Generalized estimating equations. London: Chapman and Hall/CRC.

Hinde, R. A. (1987). Individuals, relationships and culture: Links between ethology and the social sciences. Cambridge; New York: Cambridge University Press.

Jacobson, D. (1987). The cultural context of social support and support networks. Medical Anthropology Quarterly, 1(1), 42-67. doi: 10.1525/maq.1987.1.1.02a00030

Kelley, H. H., \& Thibaut, J. W. (1978). Interpersonal relations: A theory of interdependence. New York: Wiley.

Kenny, D. A., \& Kashy, D. A. (2011). Dyadic data analysis using multilevel modeling. In J. J. Hox \& J. K. Roberts (Eds.), Handbook of advanced multilevel analysis (p. 335-370). New York: Routledge.

Kenny, D. A., Kashy, D. A., \& Cook, W. L. (2006). Dyadic data analysis. Guilford Press.

Keppel, G., \& Wickens, T. D. (2004). Design and analysis: A resercher's handbook (4th ed.). Upper Saddle River, NJ: Prentice-Hall.

Kessler, R. C., Brown, R. L., \& Broman, C. L. (1981). Sex differences in psychiatric help-seeking: evidence from four large-scale surveys. Journal of health and social behavior, 22(1), 49-64. 
Kim, H. S., \& Markus, H. R. (1999). Deviance or uniqueness, harmony or conformity? A cultural analysis. Journal of Personality and Social Psychology, 77(4), 785-800. doi: 10.1037/0022-3514.77.4.785

Kim, H. S., Sherman, D. K., Ko, D., \& Taylor, S. E. (2006). Pursuit of comfort and pursuit of harmony: Culture, relationships, and social support seeking. Personality and Social Psychology Bulletin, 32(12), 1595-1607. doi: 10.1177/0146167206291991

Kim, H. S., Sherman, D. K., \& Taylor, S. E. (2008). Culture and social support. American Psychologist, 63(6), 518-526. doi: 10.1073/pnas.1010830107

Kim, M. S., Shin, H. C., \& Cai, D. (1998). Cultural influences on the preferred forms of requesting and re-requesting. Communication Monographs, 65(1), 47-66. doi: $10.1080 / 03637759809376434$

Lawrence, E., Bunde, M., Barry, R. A., Brock, R. L., Sullivan, K. T., Pasch, L. A., ... Adams, E. E. (2008). Partner support and marital satisfaction: Support amount, adequacy, provision, and solicitation. Personal Relationships, 15(4), 445-463. doi: 10.1111/j.1475-6811.2008.00209.x

Leatham, G., \& Duck, S. (1990). Conversations with friends and the dynamics of social support. In S. Duck \& R. Cohen (Eds.), Personal relationships and social support (p. 1-29). London: Sage Publications, Inc.

Loeys, T., Cook, W., De Smet, O., Wietzker, A., \& Buysse, A. (2014). The actor-partner interdependence model for categorical dyadic data: A user-friendly guide to GEE. Personal Relationships, 21(2), 225-241. doi: 10.1111/pere.12028

Loeys, T., \& Molenberghs, G. (2013). Modeling actor and partner effects in dyadic data when outcomes are categorical. Psychological Methods, 18(2), 220-236. doi: $10.1037 / \mathrm{a} 0030640$

Markus, H. R., \& Kitayama, S. (2010). Cultures and selves: A cycle of mutual constitution. Perspectives on Psychological Science, 5(4), 420-430. doi: 10.1177/1745691610375557

Martin, R., Davis, G. M., Baron, R. S., Suls, J., \& Blanchard, E. B. (1994). Specificity in 
social support: Perceptions of helpful and unhelpful provider behaviors among irritable bowel syndrome, headache, and cancer patients. Health Psychology, 13(5), 432-439. doi: $10.1037 / 0278-6133.13 .5 .432$

Masters, K. S., Stillman, A. M., \& Spielmans, G. I. (2007). Specificity of social support for back pain patients: Do patients care who provides what? Journal of Behavioral Medicine, 30 (1), 11-20. doi: 10.1007/s10865-006-9078-z

McKnight, P. E., McKnight, K. M., Sidani, S. F., \& José, A. (2007). Missing data: A gentle introduction (D. A. Kenny, Ed.). New York, NY, US: Guilford Press.

Mojaverian, T., \& Kim, H. S. (2013). Interpreting a helping hand: Cultural variation in the effectiveness of solicited and unsolicited social support. Personality and Social Psychology Bulletin, 39(1), 88-99. doi: 10.1177/0146167212465319

Muraco, A. (2012). Odd couples: Friendships at the intersection of gender and sexual orientation. Durham, NC: Duke University Press.

Neff, L. A., \& Karney, B. R. (2005). Gender differences in social support: A question of skill or responsiveness? Journal of Personality and Social Psychology, 88(1), 79-90. doi: 10.1037/0022-3514.88.1.79

Park, J., Kitayama, S., Karasawa, M., Curhan, K., Markus, H. R., Kawakami, N., ... Ryff, C. D. (2013). Clarifying the links between social support and health: Culture, stress, and neuroticism matter. Journal of Health Psychology, 18(2), 226-235. doi: $10.1177 / 1359105312439731$

Pasch, L. A., Bradbury, T. N., \& Davila, J. (1997). Gender, negative affectivity, and observed social support behavior in marital interaction. Personal Relationships, 4(4), 361-378. doi: 10.1111/j.1475-6811.1997.tb00151.x

Rubin, K. H., Bukowski, W. M., \& Parker, J. G. (2006). Peer interactions, relationships, and groups. In N. Eisenberg (Ed.), Handbook of child psychology (Vol. 3, p. 571-645). John Wiley \& Sons, Inc.

Rusbult, C. E., \& Van Lange, P. A. M. (2008). Why we need interdependence theory. Social 
and Personality Psychology Compass, 2(5), 2049-2070. doi:

10.1111/j.1751-9004.2008.00147.x

Sadler, P., Ethier, N., \& Woody, E. (2011). Tracing the Interpersonal Web of

Psychopathology: Dyadic Data Analysis Methods for Clinical Researchers. Journal of Experimental Psychopathology, 2(2), 95-138. doi: 10.5127/jep.010310

Schuster, T. L., Kessler, R. C., \& Aseltine, R. H. (1990). Supportive interactions, negative interactions, and depressed mood. American Journal of Community Psychology, 18(3), 423-438. doi: 10.1007/BF00938116

Sheppard, M. (2012). Allfitdist. Retrieved from http://www . mathworks . com/matlabcentral/

Shumaker, S. A., \& Hill, D. R. (1991). Gender differences in social support and physical health. Health, 10(2), 102-111. doi: 10.1037/0278-6133.10.2.102

Suhr, J. A., Cutrona, C. E., Krebs, K. K., \& Jensen, S. L. (2004). The social support behavior code. In P. K. Kerig \& D. Baucom (Eds.), Couple observational coding systems (p. 311-318). Mahwah, NJ: Erlbaum.

Sullivan, K. T., Pasch, L. A., Johnson, M. D., \& Bradbury, T. N. (2010). Social support, problem solving, and the longitudinal course of newlywed marriage. Journal of Personality and Social Psychology, 98(4),631-644. doi: 10.1037/a0017578

Tamres, L. K., Janicki, D., \& Helgeson, V. S. (2002). Sex differences in coping behavior: A meta-analytic review and an examination of relative coping. Personality and Social Psychology Review, 6(1), 2-30. doi: 10.1207/S15327957PSPR0601_1

Taylor, S. E. (2007). Social support. In H. S. Friedman \& R. C. Silver (Eds.), Foundations of health psychology (p. 145-171). New York: Oxford University Press.

Taylor, S. E. (2011). How psychosocial resources enhance health and well-being. In S. I. Donaldson, M. Csikszentmihalyi, \& J. Nakamura (Eds.), Applied positive psychology: Improving everyday life, health, schools, work, and society (p. 65-77). New York, NY, US: Routledge/Taylor \& Francis Group. 
Taylor, S. E., Sherman, D. K., Kim, H. S., Jarcho, J., Takagi, K., \& Dunagan, M. S. (2004). Culture and social support: Who seeks it and why? Journal of Personality and Social Psychology, 87(3), 354-362. doi: 10.1037/0022-3514.87.3.354

Verhofstadt, L. L., Buysse, A., \& Ickes, W. (2007). Social support in couples: An examination of gender differences using self-report and observational methods. Sex Roles, 57(3), 267-282. doi: 10.1007/s11199-007-9257-6

Verhofstadt, L. L., Lemmens, G. M., \& Buysse, A. (2012, De). Support-seeking, support-provision and support-perception in distressed married couples: a multi-method analysis. Journal of Family Therapy, 35(3), 320-339. doi: $10.1111 / 1467-6427.12001$

Wang, S., \& Repetti, R. L. (2014). Psychological well-being and job stress predict marital support interactions: A naturalistic observational study of dual-earner couples in their homes. Journal of Personality and Social Psychology, 107(5), 864-878. doi: $10.1037 / \mathrm{a} 0037869$

Wang, S., Shih, J. H., Hu, A. W., Louie, J. Y., \& Lau, A. S. (2010). Cultural differences in daily support experiences. Cultural Diversity and Ethnic Minority Psychology, 16(3), 413-420. doi: 10.1037/a0019885

Wickham, R. E., \& Knee, C. R. (2012). Interdependence theory and the actor-partner interdependence model: Where theory and method converge. Personality and Social Psychology Review, 16(4), 375-393. doi: 10.1177/1088868312447897

Wilhelm, K. A. (2014). Gender and mental health. Australian \& New Zealand Journal of Psychiatry, 48(7), 603-605. doi: 10.1177/0004867414538678

Wills, T. A. (1991). Social support and interpersonal relationships. In M. S. Clark (Ed.), Prosocial behaviours (p. 265-289). Thousand Oaks, CA, US: Sage Publications, Inc.

Wonderlich-Tierney, A. L., \& Vander Wal, J. S. (2010). The effects of social support and coping on the relationship between social anxiety and eating disorders. Eating Behaviors, 11(2), 85-91. doi: 10.1016/j.eatbeh.2009.10.002 
Zimet, G. D., Dahlem, N. W., Zimet, S. G., \& Farley, G. K. (1988). The multidimensional scale of perceived social support. Journal of Personality Assessment, 52(1), 30-41. doi: 10.1207/s15327752jpa5201_2 


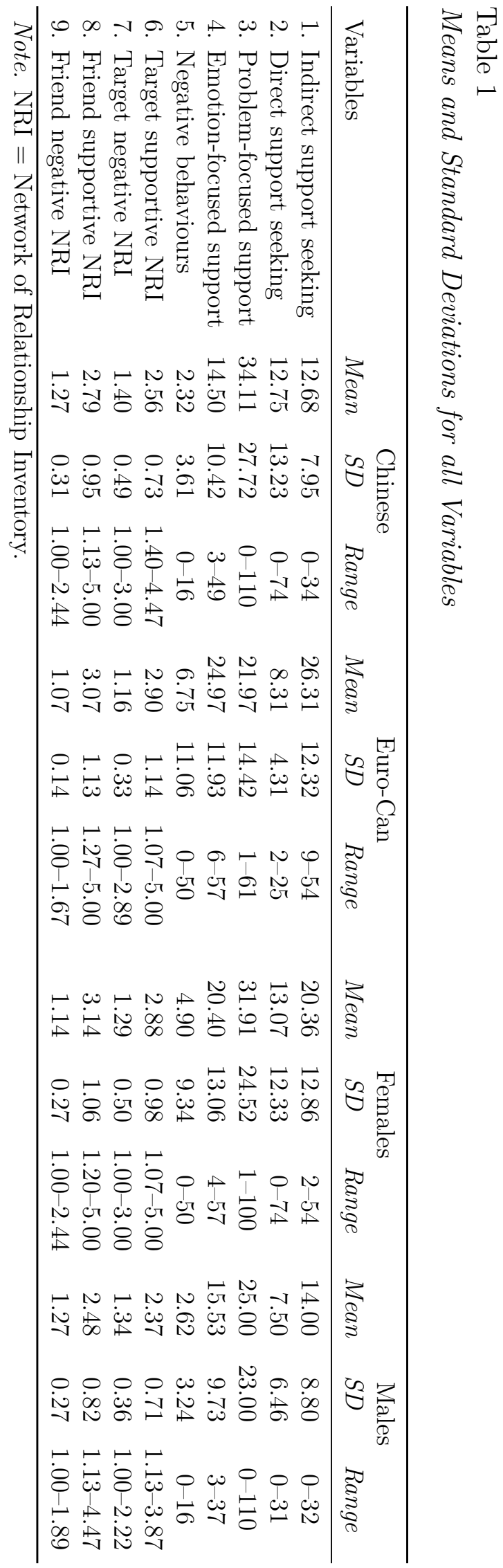


Table 2

Correlations for all Variables

\begin{tabular}{|c|c|c|c|c|c|c|c|c|c|}
\hline Variables & 1 & 2 & 3 & 4 & 5 & 6 & 7 & 8 & 9 \\
\hline 1. Indirect support seeking & & $0.54^{* *}$ & * $0.36^{* *}$ & $0.58^{* *}$ & $* 0.13$ & $-0.09-$ & -0.17 & 0.22 & -0.05 \\
\hline 2. Direct support seeking & $0.38^{*}$ & & $0.70^{* *}$ & $0.75^{* *}$ & * 0.06 & $0.05-$ & -0.02 & 0.17 & -0.10 \\
\hline 3. Problem-focused support & $0.44^{* *}$ & $* 0.57 * *$ & & $0.61^{* *}$ & $* 0.21$ & 0.05 & 0.01 & 0.10 & -0.09 \\
\hline 4. Emotion-focused support & 0.30 & $0.51^{* *}$ & $* 0.58 * *$ & & -0.06 & $-0.01-$ & -0.21 & 0.13 & -0.06 \\
\hline 5. Negative behaviours & 0.23 & $0.60^{* *}$ & $* 0.62^{* *}$ & 0.21 & & -0.02 & 0.15 & -0.14 & 0.03 \\
\hline 6. Target supportive NRI & 0.003 & 0.14 & 0.05 & $0.21-$ & -0.01 & & $0.40^{* *}$ & * $0.29 *$ & 0.15 \\
\hline 7. Target negative NRI & -0.13 & -0.06 & -0.01 & $0.05-$ & -0.04 & 0.19 & & 0.03 & 0.20 \\
\hline 8. Friend supportive NRI & 0.002 & $0.39^{*}$ & 0.15 & $0.35^{*}$ & 0.16 & $0.84^{* *}$ & $* 0.09$ & & 0.13 \\
\hline 9. Friend negative NRI & -0.14 & -0.26 & -0.02 & -0.02 & 0.04 & -0.12 & 0.07 & -0.20 & \\
\hline
\end{tabular}

Note. Upper diagonal presents correlation coefficients for Chinese participants while the lower diagonal presents correlation coefficients for Euro-Canadians participants. NRI $=$ Network of Relationship Inventory. 
Table 3

Cultural and Gender Variations Using Generalized Linear Models

\begin{tabular}{|c|c|c|c|c|c|}
\hline Dependent Variables & $B$ & $S E$ & $95 \% C I$ & $p$-value & $\operatorname{Exp}(B)$ \\
\hline
\end{tabular}

Independent Variable: Cultural Contexts

1. Indirect support seeking $\quad \begin{array}{rrrrr}0.73 & 0.11 & {[0.51,0.95]} & 0.000 & 2.08\end{array}$

2. Direct support seeking $\quad-0.43 \quad 0.16 \quad[-0.75,-0.11] \quad 0.008 \quad 0.65$

3. Problem-focused support $\quad-0.44 \quad 0.16 \quad[-0.74,-0.14] \quad 0.004 \quad 0.64$

4. Emotion-focused support $\quad \begin{array}{llllll}0.54 & 0.12 & {[0.30,0.79]} & 0.000 & 1.72\end{array}$

$\begin{array}{llllll}\text { 5. Negative behaviours } & 1.07 & 0.34 & {[0.40,1.73]} & 0.002 & 2.91\end{array}$

Independent Variable: Gender

1. Indirect support seeking $\quad-0.38 \quad 0.13 \quad[-0.64,-0.11] \quad 0.005 \quad 0.69$

2. Direct support seeking $\quad-0.56 \quad 0.19 \quad[-0.93,-0.18] \quad 0.004 \quad 0.57$

3. Problem-focused support $\quad-0.24 \quad 0.18 \quad\left[\begin{array}{lllll}-0.61,0.12 & 0.187 & 0.78\end{array}\right.$

4. Emotion-focused support $\quad-0.27 \quad 0.13 \quad\left[\begin{array}{llll}-0.54,-0.01 & 0.043 & 0.76\end{array}\right.$

$\left.\begin{array}{llllll}5 . & \text { Negative behaviours } & -0.63 & 0.32 & {[-1.26,0.01}\end{array}\right] \quad 0.054 \quad 0.54$

Note. Chinese cultural context was coded as 1 and Euro-Canadian cultural context was coded as 0; Female was coded as 1 and male was coded as 0 in these sets of analyses. 


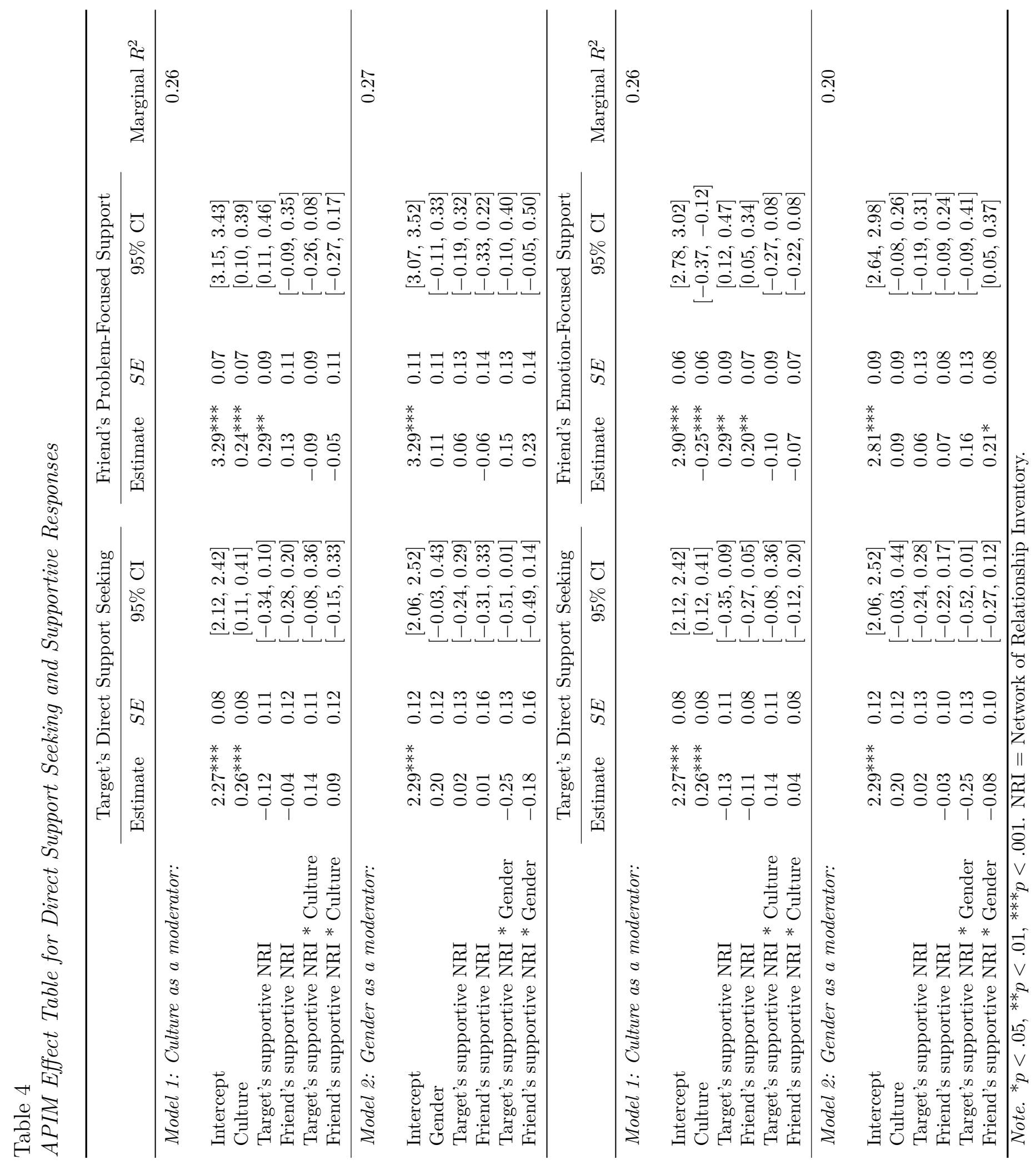




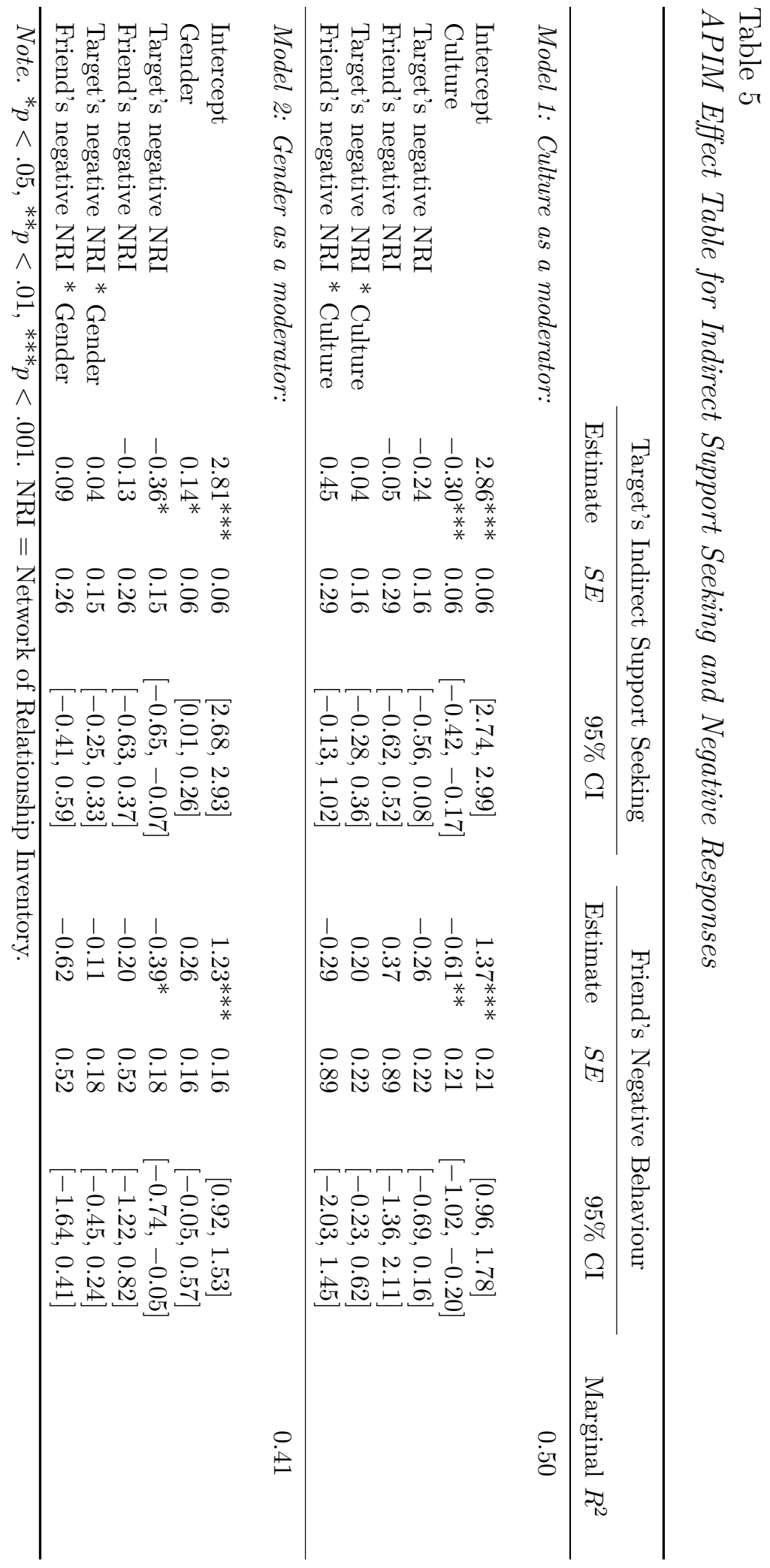




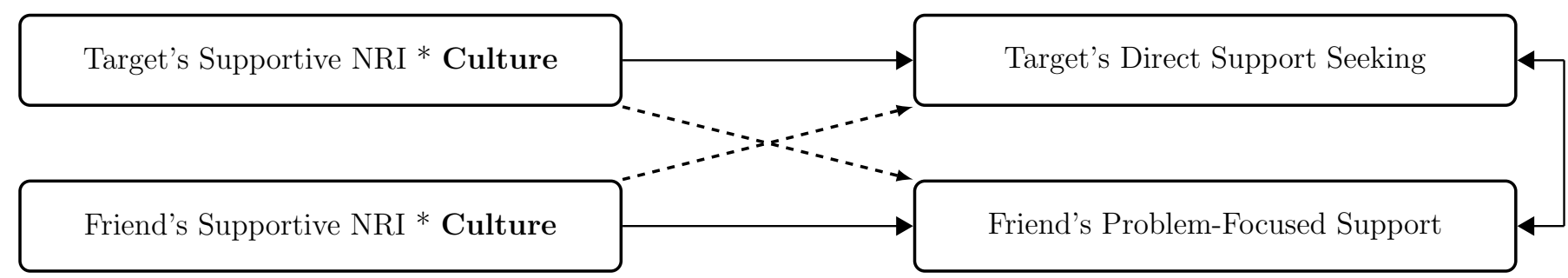

(a) Actor-Partner Interdependence Model 1.

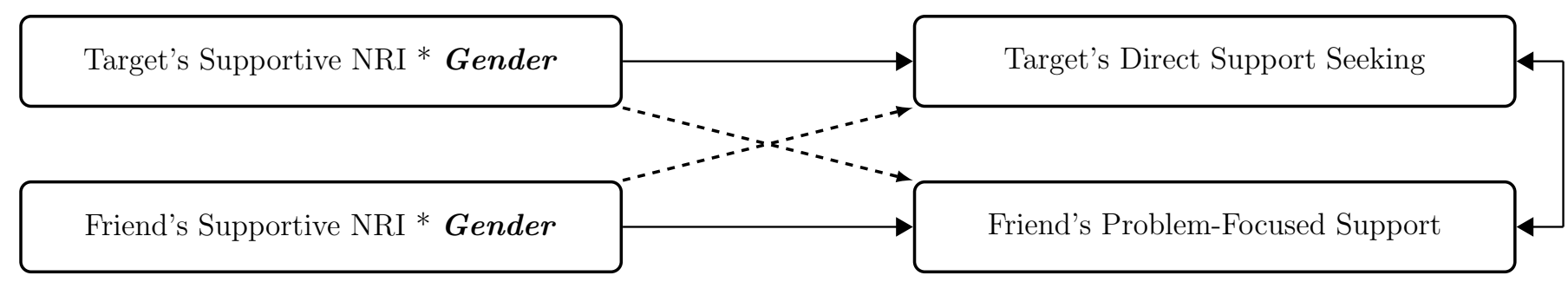

(b) Actor-Partner Interdependence Model 2.

Figure 1. Actor-Partner Interdependence Models with three-way interactions for direct support seeking and problem-focused support. 


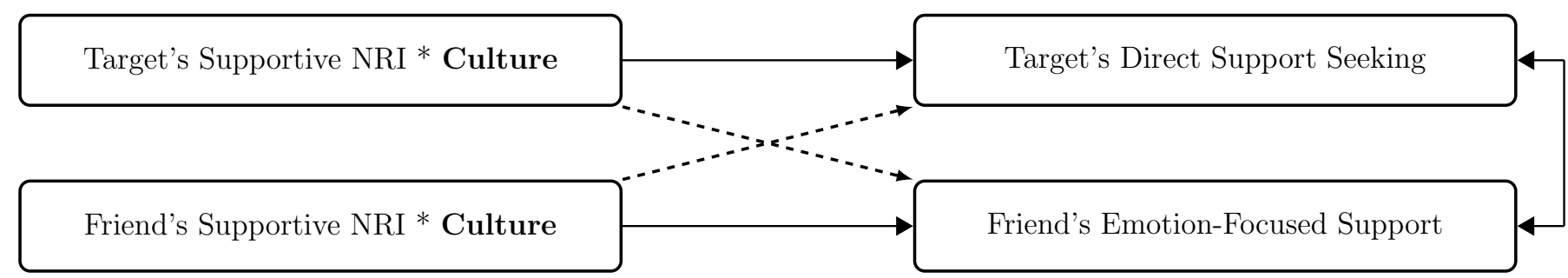

(a) Actor-Partner Interdependence Model 1.

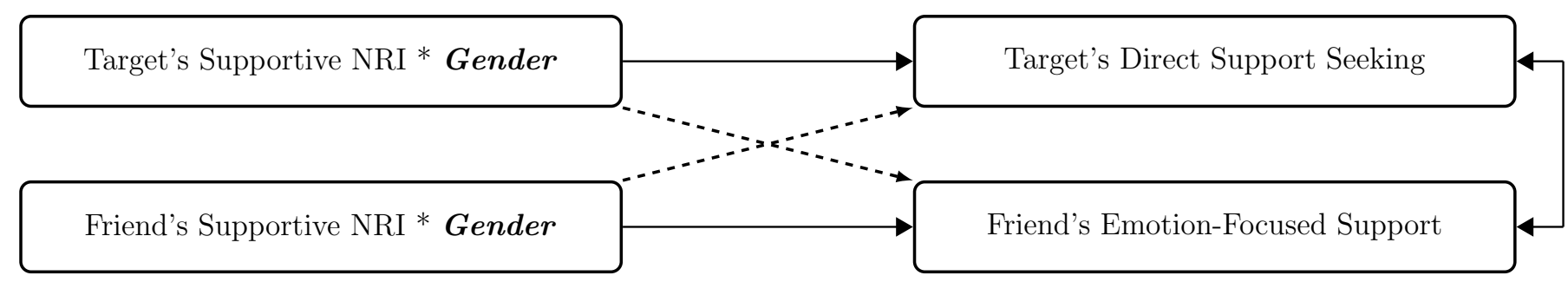

(b) Actor-Partner Interdependence Model 2.

Figure 2. Actor-Partner Interdependence Models with three-way interactions for direct support seeking and emotion-focused support. 


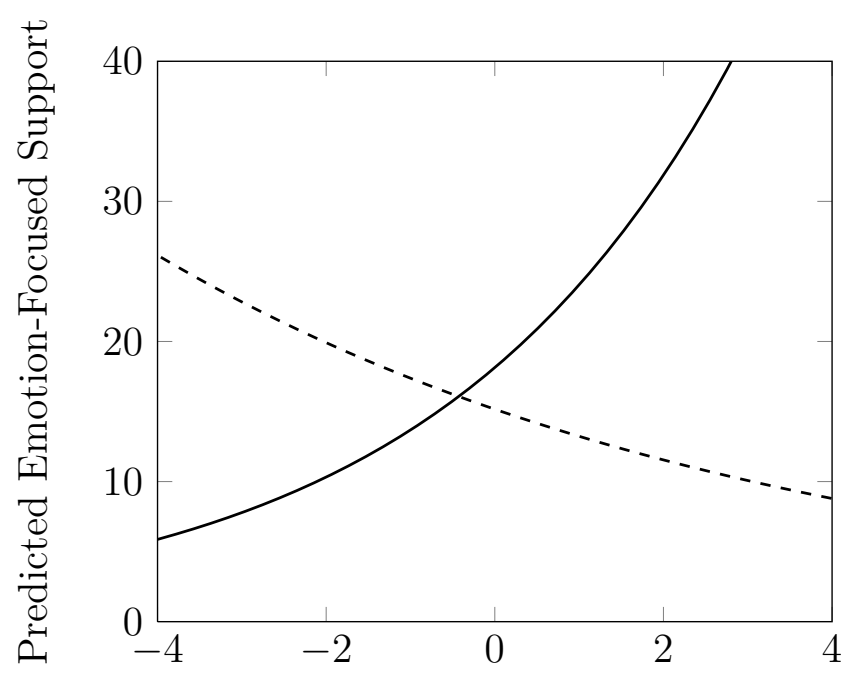

(a) Mean Friend's Supportive Friendship

Figure 3. Gender variations in the Friend's actor effect (Females —, Males -- - ). 


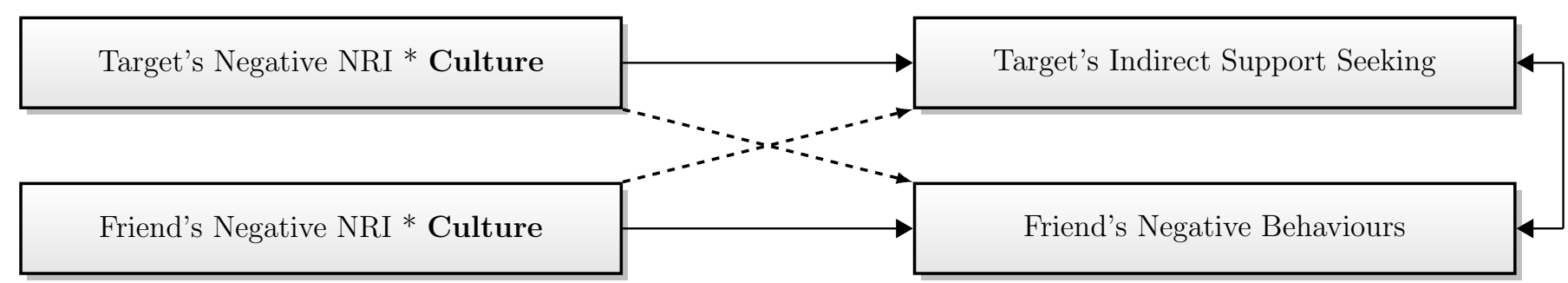

(a) Actor-Partner Interdependence Model 1.

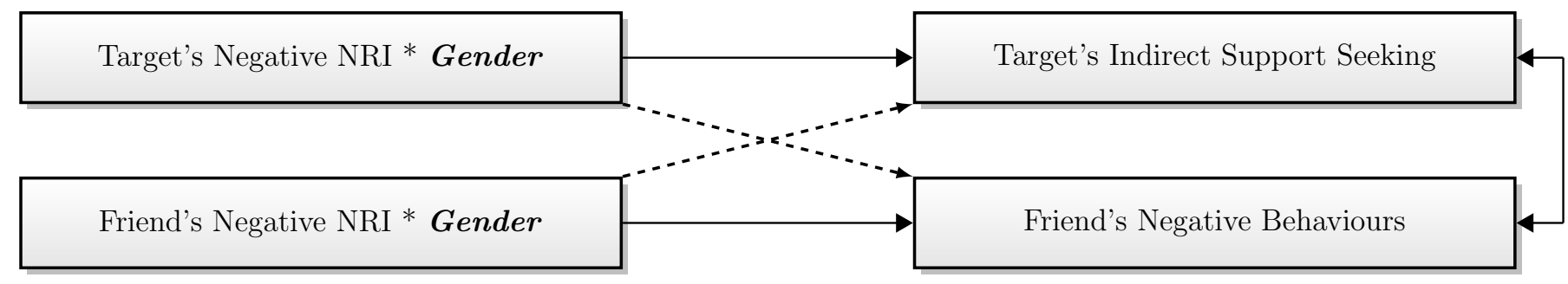

(b) Actor-Partner Interdependence Model 2.

Figure 4. Actor-Partner Interdependence Models with three-way interactions for indirect support seeking and negative behaviours. 
Appendix

Equations for the two-intercept model using Culture as the moderator

The two intercept model contains two equations - one for the Target and one for the Friend: Equation for the Target:

$$
\begin{aligned}
\log \left(\operatorname{Pr}\left(Y_{i j}\right)\right) & =\beta_{0 T} \text { Target }+\beta_{1 T} \text { Target } * \text { Culture } \\
& +\beta_{2 T} \text { Target } * \text { SupportiveN RI } I_{\text {actor }} \\
& +\beta_{3 T} \text { Target } * \text { SupportiveN RI } I_{\text {partner }} \\
& +\beta_{4 T} \text { Target } * \text { SupportiveN } R I_{\text {actor }} * \text { Culture } \\
& +\beta_{5 T} \text { Target } * \text { SupportiveN } R I_{\text {partner }} * \text { Culture }
\end{aligned}
$$

Equation for the Friend:

$$
\begin{aligned}
\log \left(\operatorname{Pr}\left(Y_{i j}\right)\right) & =\beta_{0 F} \text { Friend }+\beta_{1 F} \text { Friend } * \text { Culture } \\
& +\beta_{2 F} \text { Friend } * \text { SupportiveNRI } I_{\text {actor }} \\
& +\beta_{3 F} \text { Friend } * \text { SupportiveN } R I_{\text {partner }} \\
& +\beta_{4 F} \text { Friend } * \text { SupportiveN } R I_{\text {actor }} * \text { Culture } \\
& +\beta_{5 F} \text { Friend } * \text { SupportiveN } R I_{\text {partner }} * \text { Culture }
\end{aligned}
$$

The actor effect of Target's supportive friendship may be presented as:

$$
\left(\beta_{2 T}+\beta_{4 T} * \text { Culture }\right) * \text { Target } * \text { SupportiveN } R I_{\text {actor }}
$$

Similarly, the actor effect of Friend's supportive friendship may be presented as:

$$
\left(\beta_{2 F}+\beta_{4 F} * \text { Culture }\right) * \text { Friend } * \text { Supportive } N R I_{\text {actor }}
$$

The partner effects of Target's and Friend's supportive friendship can be written as:

$$
\left(\beta_{3 T}+\beta_{5 T} * \text { Culture }\right) * \text { Target } * \text { SupportiveN } R I_{\text {partner }}
$$

and

$$
\left(\beta_{3 F}+\beta_{5 F} * \text { Culture }\right) * \text { Friend } * \text { SupportiveNRI } I_{\text {partner }}
$$


Because Culture was coded as Chinese $=1$ and Euro-Canadians $=-1$, Equation (3) to Equation (6) yield:

For Chinese Target's actor effects:

$$
\left(\beta_{2 T}+\beta_{4 T}\right) * \text { Target } * \text { SupportiveNRI } I_{\text {actor }}
$$

For Chinese Friend's actor effects:

$$
\left(\beta_{2 F}+\beta_{4 F}\right) * \text { Friend } * \text { SupportiveN RI } I_{\text {actor }}
$$

For Euro-Canadian Target's actor effects:

$$
\left(\beta_{2 T}-\beta_{4 T}\right) * \text { Target } * \text { SupportiveNRI } I_{\text {actor }}
$$

For Euro-Canadian Friend's actor effects:

$$
\left(\beta_{2 F}-\beta_{4 F}\right) * \text { Friend } * \text { SupportiveNRI } I_{\text {actor }}
$$

Therefore, Chinese partner effects are:

$$
\begin{aligned}
& \left(\beta_{3 T}+\beta_{5 T}\right) * \text { Target } * \text { SupportiveN RI } I_{\text {partner }} \\
& \left(\beta_{3 F}+\beta_{5 F}\right) * \text { Friend } * \text { SupportiveN RI } I_{\text {partner }}
\end{aligned}
$$

Euro-Canadian partner effects are:

$$
\begin{aligned}
& \left(\beta_{3 T}-\beta_{5 T}\right) * \text { Target } * \text { SupportiveN RI } I_{\text {partner }} \\
& \left(\beta_{3 F}-\beta_{5 F}\right) * \text { Friend } * \text { SupportiveN RI } I_{\text {partner }}
\end{aligned}
$$

In sum, the results for Target's actor effect are: with one unit increase in supportive interactions, there will be $\exp \left(\beta_{2 T}+\beta_{4 T}\right)$ change for Chinese Target and $\exp \left(\beta_{2 T}-\beta_{4 T}\right)$ for Euro-Canadian Target. Following the same logic, the results for Friend's partner effect are: with one unit increase in supportive interactions, there will be $\exp \left(\beta_{3 F}+\beta_{5 F}\right)$ change for Chinese Friend and $\exp \left(\beta_{3 F}-\beta_{5 F}\right)$ change for Euro-Canadian Friend. 